\title{
Global acid and metalliferous drainage management standard: BHP's approach to reducing global acid and metalliferous drainage closure risk
}

\author{
JP Pearce Mine Waste Management, Australia \\ T Cooper BHP, Australia
}

J Heyes BHP, Australia

\begin{abstract}
BHP operates mines across the globe that, along with providing minerals required for societal development, also generate mine waste rock. This waste rock and exposed rock surfaces could potentially result in acid and metalliferous drainage (AMD) if the operations and materials are not properly identified and managed. Because AMD can occur long beyond the operational life of a mine, AMD risk can be a key risk driver in closure cost estimates. Therefore, to address this risk, BHP has developed a global AMD management standard to be implemented across all BHP's functions and operations, including closed sites.
\end{abstract}

The purpose of the AMD management standard is to provide a management framework to support consistent, simple and sustainable global management of AMD risks. This in turn is designed to assist the reduction of uncertainty in closure cost estimates. Compliance to the AMD management standard informs several elements of BHP's closure management process, specifically baseline data and knowledge, risk assessment and ongoing implementation and review of Closure Management Plans.

The AMD management framework, from which the standard is based, was adapted from industry bestpractice guidance to ensure its applicability to multiple regions and commodities. The AMD Management Framework consists of sequential requirements throughout the mining lifecycle from early mine studies, mine planning, mine development and operations, through to closure and throughout post-closure. The framework is iterative with an adaptive management approach designed to incorporate new data/information and management opportunities into AMD risk assessments and mine plan revisions.

To promote the effective implementation and adoption of the standard, interactive consultation was undertaken with various groups across BHP's assets. This early consultation enabled key concerns to be raised and addressed, which is designed at promoting ownership of the AMD management standard. This paper discusses the framework to which BHP's AMD management standard is based and provides an outline for its planned implementation.

Keywords: acid and metalliferous drainage, $A M D, A M D$ management, $A M D$ risk

\section{Introduction}

Acid and metalliferous drainage (AMD) is recognised internationally as one of the most difficult and significant environmental issues facing both miners and regulatory bodies in the pursuit of a sustainable mining industry. This significance is due to the ability of AMD pollution to persist for hundreds, if not thousands of years (Pearce et al. 2016a). There are numerous international examples where this ability to persist many years after the cessation of mining operations is demonstrated. For instance, Australian examples include the Mt Morgan Mine in Queensland, which is predicted to generate AMD for up to 500 years (Gasparon et al. 2007) and the rehabilitation of the Rum Jungle Mine in the Northern Territory, which is expected to be in the hundreds of millions of dollars. The Iron Mountain Superfund site in California, which ceased mining in the early 1960s is responsible for AMD discharging into the Sacramento River with 
suspected fish kills as early as 1939 (Nordstrom \& Alpers 1999). The Richmond Mine orebody, which is one of the Iron Mountain group of mines, still contains enough pyrite within the deposit to continue oxidising for more than 3,000 years (Nordstrom and Alpers 1999).

The need to adequately manage potentially AMD generating materials has driven the development of numerous AMD related guidance documents (e.g. Commonwealth of Australia 2016a; Department of Mines and Petroleum 2015; Sernageomin 2015). These documents often set minimum guidelines for AMD management. To achieve a sustainable mining industry with respect to potentially AMD generating material, these minimum guidelines must be used in combination with other key industry standard sources such as the Global Acid Rock Drainage (GARD) Guide (INAP, 2009) and the Prediction Manual for Drainage Chemistry from Sulphidic Geologic Materials (Price, 2009) to develop industry best-practice approaches that addresses both current and future social licences.

BHP operates mines across the globe that, along with providing minerals required for societal development, also generate mine waste rock. This waste rock and exposed rock surfaces could potentially result in AMD if the operations and materials are not properly identified and managed. Once AMD impacted waters are generated, they are difficult to manage and can represent a significant portion of a site's closure liability. Recognising this risk to closure, a global AMD management standard (the Standard) has been developed that outlines minimum requirements for consistent and practicable AMD management across all BHP's functions and operations, including closed sites. The Standard describes AMD management objectives, requirements and supporting documents upon which the Standard is based.

\section{$2 \quad$ Acid and metalliferous drainage}

The processes involved in generating and releasing AMD are well described (Lottermoser 2010; Miller 2014; Pearce et al. 2016a). Due to BHP's global span, it is important to clearly define AMD as the acronym can have various meanings in different regions. BHP have adopted the South Pacific nomenclature (Price, 2009) for AMD which defines it as 'acid and metalliferous drainage'. In this definition, AMD includes the release of low $\mathrm{pH}$ drainage waters otherwise described as acid rock drainage (ARD) from potentially acid forming (PAF) mine waste or exposed surfaces. It can also include drainage containing high metals or salinity in non-acidic waters, such as neutral metalliferous drainage (NMD) and/or saline drainage (SD).

\section{History of BHP acid and metalliferous drainage management, the Western Australia iron ore journey}

Each year, iron ore mine operators in the Pilbara move significantly more mineral waste material than saleable product, creating landforms and potential liabilities that will remain after the operating mine life, several of which will contain potentially AMD generating materials. Therefore, AMD risk across Western Australian iron ore mines, collectively represents one of the key closure and operational environmental management liabilities.

To address this risk, BHP developed a consistent and practicable AMD Management System that spans across all Western Australian Iron Ore (WAIO) functions and operations. The AMD Management System ensures that AMD risk is consistently identified, managed and controlled throughout the life-of-mine from exploration through mine planning, operations and closure. The development, implementation and adoption of the AMD Management System has been a long journey for BHP that now sees it recognised as industry leaders in AMD management.

Previously, within BHP the understanding of AMD risk across Pilbara mines was limited to a small group of people. This led to AMD risk in BHP's WAIO operations to being both potentially over- and under-managed by individual sites without consistency across the Pilbara operations. These shortcomings were identified internally and a concentrated long-term effort was initiated to address these areas for improvement.

At the beginning of WAIO's six year journey was the development of a WAIO AMD Management Standard (the WAIO Standard). The WAIO Standard outlines AMD management requirements for the life-of-mine to 
ensure that risks associated with AMD are identified and controlled. It is a multi-faceted approach that incorporates a range of activities by WAIO functions and operations to enable effective AMD management. Following on from the WAIO Standard was the development of detailed guidelines for waste management as well as an AMD risk assessment procedure with associated tools. Supporting the implementation and adoption of these guidelines and procedures was an ongoing WAIO-wide AMD risk education program. This program included annual short-courses being provided to personnel from multiple sites and disciplines (including mine planners, production managers, environment specialists, geologists and hydrogeologists). The AMD basics short-course is aimed at developing a greater awareness of AMD risk while educating those responsible for implementing various aspects of the WAIO Standard. The outcome of this approach to AMD management within the company is that WAIO are now leading the example for other assets to follow (Figure 1).

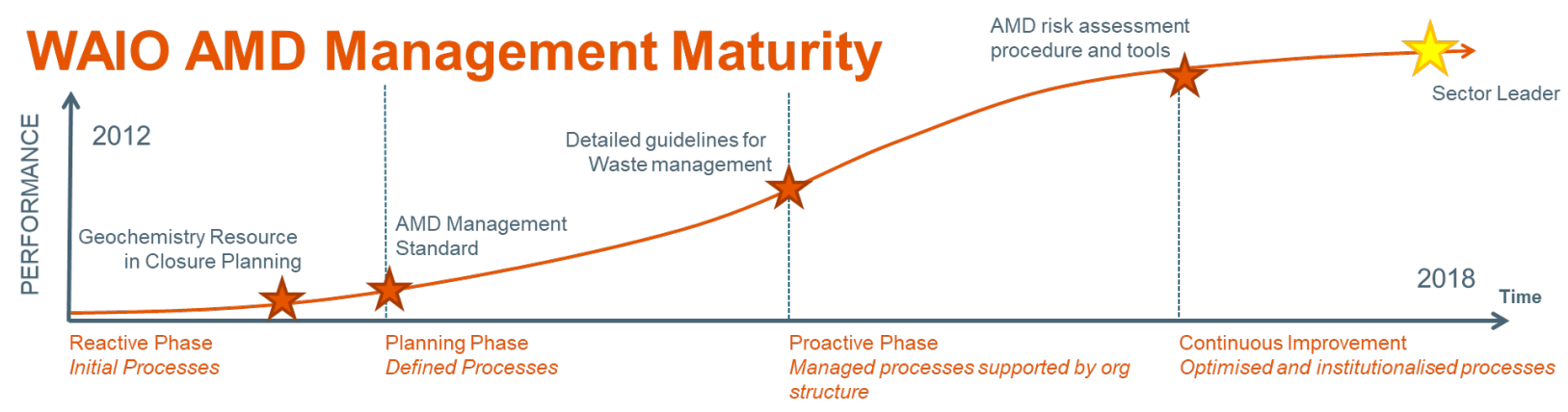

Figure 1 An acid and metalliferous drainage (AMD) management maturity curve that demonstrates Western Australian Iron Ore's (WAIO) journey. This maturity curve is used as a differentiator for assets within the company to comparatively view the various degrees of AMD management maturity

\section{$4 \quad$ Global acid and metalliferous drainage management standard}

Drawing from WAIO's success in reducing both their risk and uncertainty with respect to AMD management, which has also reduced uncertainty around closure liabilities, BHP developed a global AMD management standard. This standard states BHP's overall AMD management strategy (the AMD Strategy) and then details the framework for which the AMD Strategy can be executed. Compliance to the Standard informs several elements of BHP's closure management process, specifically baseline data and knowledge, risk assessment and ongoing implementation and review of closure management plans.

\subsection{Acid and Metalliferous Drainage Management Strategy}

Our understanding of how waste management methods impact the generation and subsequent release of AMD has significantly improved over the past 20 years (e.g. Wilson 2011; Miller 2014; Pearce et al., 2016b). However, this better understanding of how waste management influences the generation and release of AMD from source facilities has not been coupled with an equal improvement in waste management itself, across the industry. A key factor in this is the perceived difficulty with executing preventative AMD management methods.

For example, segregation of waste rock so that high-risk material can be managed to inhibit oxidation occurring (preventative measure) has the perception that this will automatically be associated with greater haulage costs and/or potential delays to production. However, if waste rock classification systems are inbuilt into the short-term mining and don't require testing of materials during grade-control drilling and sampling to classify waste blocks, waste can be moved without delay, provided QA/QC processes are in place to validate the approach (performance monitoring). In some cases, optimising long-term waste haulage routes over the life of the mine can also result in lower total haulage costs than compared with haulage routes optimised for the short-term. 
Another example is the perceived difficulties in constructing complex oxygen reducing layers within a waste rock dump (WRD) to inhibit oxidation occurring (preventative measure). Although past designs of oxygen inhibiting structures may be more akin to civil construction projects, simple thin lift construction methods can easily be executed with available mining equipment. These perceived difficulties subsequently lead to the continued selection of mitigation and control and treat methods, which is often the wrong approach for a sustainable mining industry.

Over WAIO's AMD management journey, BHP have been able to work through these perceptions and change the way they approach AMD management. This site-based evidence of successful execution of preventive AMD management methods has been the driver for the development of the AMD Strategy.

BHP's overarching AMD Strategy is that prevention (source control) of AMD rather than treatment in perpetuity, is the preferred lowest risk approach. The AMD Strategy is obviously easier to execute at operational mines in comparison to legacy sites where waste has already been placed and water collection and treatment systems are established. However, this preferred management approach is still assessed for these legacy sites and will drive future research and development to facilitate the movement of these sites towards source control, where possible.

The AMD Strategy incorporates three key components, compliance, responsibility and sustainability, which are garnering support from industry as the lower risk sustainable pathway for modern mining (Weber et al. 2019). Compliance speaks to the regulatory commitments associated with each mining licence; these are considered the minimum requirements. Responsibility refers to BHP's current social licence, which should be met by adopting best practicable AMD management approaches. Sustainability represents BHP's future social license that may be associated with changing society expectations (generational and emerging risks). Adopting a prevention (source control) focused AMD Strategy addresses the sustainability component.

\subsection{Acid and Metalliferous Drainage Management Framework}

BHP's AMD Management Framework (Figure 2) is adapted from industry best-practice (Commonwealth of Australia 2016a; INAP 2007; Price 2009; Sernageomin 2015), which is underpinned by six key steps of AMD management. The six steps are Closure Goals, Prediction, Prevention, Minimisation, Control and Treat, and Performance Monitoring. The setting of closure management plan objectives, which is the first step of BHP's closure management process (Our Requirements Closure), is also the first step of AMD management.

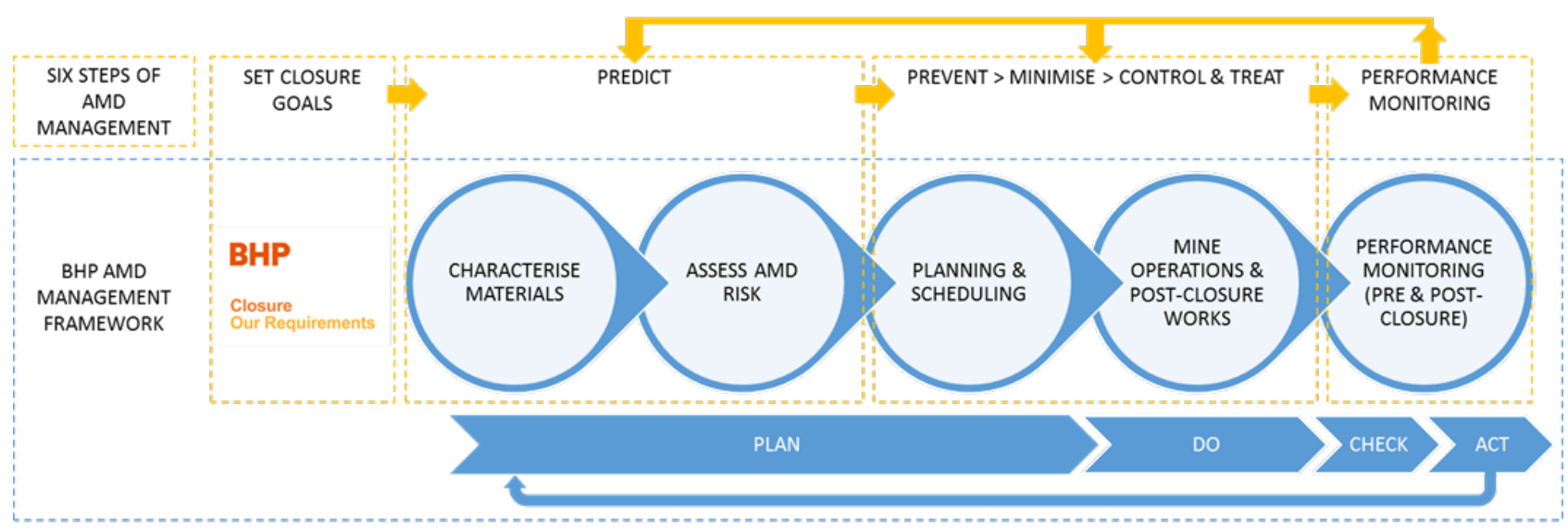

Figure 2 How BHP's Acid and Metalliferous Drainage (AMD) Management Framework links with the six steps of AMD management

The framework, which utilises a Plan-Do-Check-Act (PDCA) approach to facilitate continuous improvement, consists of sequential requirements throughout the mining lifecycle from early mine studies, mine planning, mine development and operations, through to closure and throughout post-closure. Effective PDCA cycles are essential for successful AMD management. They provide a rigorous process for reconciling actual 
performance against the plan as well as identifying and implementing changes to either recover to the original plan, or improve on the original plan as a continuous improvement process. This inbuilt PDCA approach within the framework is what facilitates adaptive management designed to incorporate new information and new technologies (research and development) into AMD risk assessments, mine plan revisions and closure plan updates.

\subsubsection{Characterise materials}

Potential AMD sources include any materials such as waste rock, ore, low-grade/mineralised waste stockpiles, pit wall/floor rock, underground workings' wall rock, tailings, process residue and heap leach materials that have the potential to generate problematic mine drainage. All potential AMD sources are identified by the development of an early stage AMD Conceptual Site Model (CSM) prior to being characterised by static and kinetic geochemical characterisation assessments to understand the geochemical nature of the materials.

The early stage AMD CSM is developed to provide an initial fundamental view of AMD potential. It is essentially a potential AMD source model with pathways and receptors to be incorporated into the subsequent $A M D$ risk assessment phase.

The specific minimum requirements for sample identification, collection and characterisation include:

- Identify potential AMD sources by developing an early stage AMD CSM.

- Where ongoing drilling programs are available (e.g. operational sites), select appropriate drilling programs to request samples in conjunction with other relevant BHP standards, e.g. Geoscience Activity Planning.

- Where ongoing drilling programs are unavailable (e.g. legacy sites, or legacy landforms), a riskbased approach should be used when deciding appropriate sampling methods.

- Static and kinetic geochemical testing programs as well as physical testing programs should be completed with parameter selections linked with other relevant BHP standards, e.g. Geoscience Data Collection, Processing and Analysis.

- Maintain an AMD data management system that interfaces with geological and assay data, located on an enterprise-wide database.

An AMD Management Standard Guidance Note was developed by BHP to provide additional details for the execution of the framework. This includes additional details such as recommended sampling frequencies and laboratory testing parameters.

\subsubsection{Assess acid and metalliferous drainage risk}

AMD risks are assessed through source definition (characterisation of potential AMD sources), identification of pathways and potential receptors. The early stage AMD CSM developed prior to the Characterisation of Potential AMD Sources is refined at this stage within the framework to incorporate pathways and receptors.

The specific minimum requirements include:

- Refine the AMD CSM and complete a preliminary assessment of potential AMD source materials. This should be made possible by the collection of data in the previous stage.

- Assess the mobilisation risk (including modelling where necessary) from potential AMD sources.

- Assess potential AMD sources and mobilisation risk with respect to protecting key receptors. That is, assess the risk in the context of a source-pathway-receptor model using the CSM as the template for this assessment. 
- To ensure AMD risk is understood and incorporated into related planning, findings of the AMD risk assessments are communicated to inform water planning, operational management strategies and closure planning.

- At the completion of the AMD risk assessment, the AMD CSM is refined based on results of the AMD risk assessment, additional characterisation studies and geochemical modelling, water management strategies, climate change assessments and closure objectives.

To ensure all BHP's risks associated with AMD are assessed, specific consideration to reputation and closure value risk is given outside the confines of a typical source-pathway-receptor model. That is, in the absence of a pathway or receptor, does the generation of AMD within a source have the potential to impact BHP's reputation or the closure value of a site, have generational and/or emerging risks been considered?

For example, if a known AMD source is stored within a WRD in a method that will allow the generation of $A M D$, are there any foreseeable impacts to future closure value such as alternative land uses? If there are, in the absence of a pathway or receptor, it may be deemed necessary to construct the WRD in a method to limit oxidation (e.g. AMD generation).

\subsubsection{Planning and scheduling}

At this stage of the AMD Framework, plans, procedures and designs for operational and post-closure works to manage AMD risks are developed. As per the hierarchy of controls and BHP's overarching AMD Strategy, preference is given to prevention strategies where achievable.

The specific requirements include:

- Estimate the quantity and schedule of all potential AMD sources in-mine plans through the life of asset. For this to be completed, AMD classifications (AMD Class 1, AMD Class 2 etc.) are developed and incorporated into mining models for effective AMD management planning.

- Where possible, avoid potential AMD sources through modification of pit and/or underground designs and waste scheduling. Another way to avoid disturbing potential AMD sources is the adjustment of drill and blast practices.

- Selectively manage AMD sources that cannot be avoided. This is done through the embedded AMD classification systems within mining models.

- For sites where sub-aqueous storage of AMD sources is possible, final storage designs and placement techniques are to consider both AMD generation rate and the time between placement and inundation. That is, if the time to inundation is a significant period of time due to the slow rebound of the groundwater table post-mining, then some preventive oxidation management methods may be required (e.g. thin lifts) to manage oxidation of sulphides between the time of placement and the time of groundwater rebound.

- For sub-aerial storage of AMD sources, final landform designs are to be developed based on principles that prevent or minimise AMD risk. Designs however must be achievable with the characteristics of the available materials, both temporally and spatially.

\subsubsection{Mine operations and post-closure works}

Operational and post-closure construction procedures are developed to verify that AMD risks are being properly managed to achieve the AMD Strategy. What procedures are developed and implemented at an individual site are dependent on the specific management methods employed at that site and will be reflective of the AMD risk for the site. 
General AMD management procedures that may be included are:

- Ongoing geochemical testing - the purpose of this procedure is to:

- Collect geochemical data on potential AMD sources and geochemical nature of the sources to allow ongoing refinement of the AMD classifications and verification of the mining model.

- Collect geochemical data and ensure QA/QC procedures are appropriate so that if required, a site can demonstrate with an acceptable degree of certainty the characteristics of materials placed in specific mine domains.

- $A M D$ classification refinement - the purpose of this procedure is to:

- Facilitate continuous refinement of a site's AMD classifications by incorporating data collected under the ongoing geochemical testing procedure, or through other geochemical studies.

- Continuously refine AMD classifications not only to ensure correct management of high-risk material but also to ensure low-risk waste rock is not incorrectly handled, which can result in unnecessary higher operational costs.

- Mining model verification - the purpose of this procedure is to:

- Provide continuous assessment of the accuracy of the mining model and the AMD classes through operational geochemical testing and refinement of the mining model.

- Check and validate the AMD classes to ensure the correct placement of potential AMD sources.

- Materials movements tracking - the purpose of this procedure is to:

- Ensure the correct handling of different types of potential AMD sources.

- Establish appropriate material movements records for regular reporting purposes.

- Minimise the amount of re-handling.

- Materials placement and compliance to plan - the purpose of this procedure is to:

- Ensure the correct management of all potential AMD sources excavated during mining and correct construction of AMD management supporting structures within the specific landform, such as lift heights, cover thicknesses etc.

- Minimise the exposure of sulphidic waste rock to water and oxygen (e.g. minimise the oxidation of sulphides and mobilisation of oxidation products - formation and release of AMD).

Each procedure developed should include a Trigger Action Response Plan (TARP) that defines the actions to be taken when conditions change from normality and have the potential to trigger an event. Primarily, TARPs should be developed for an AMD incident and include the key parameters that would facilitate the identification of that specific AMD incident occurring. A TARP will identify a series of hazard levels (triggers) for an operation that will range from normal to extremely abnormal. Each level will then be associated with a range of controls and actions, together with responsibilities for taking the appropriate actions. Regular reviews of the risk assessments and of research and monitoring will ensure triggers and planned action within the TARPs are appropriate.

\subsubsection{Performance monitoring (pre- and post-closure)}

A key aspect of performance evaluation, which assesses the response of a system while referencing specific criteria, is the ability to provide early warning of developing trends (triggers for change management) as well as facilitating the reporting of environmental performance to regulators and stakeholders (Commonwealth of Australia 2016b). Performance data also provides supporting evidence that the AMD management procedures proposed were followed and were successful, providing confidence to regulators and stakeholders that closure objectives proposed are achievable. Therefore, the overall performance of 
potential AMD source management is to be assessed by monitoring both leading and lagging indicators to validate AMD predictions and the performance of management strategies.

The specific requirements include:

- Develop a performance evaluation process to provide early warning of developing trends (triggers for change management) such as monitoring real-time parameters, e.g. in situ oxygen and temperature in WRDs and tailings storage facilities (TSFs).

- Monitor surface and groundwater quality according to water management strategies throughout mine operation and throughout the post-closure period to assess the effectiveness of AMD source management.

- Complete ongoing AMD assessments throughout the life of asset to confirm predictions regarding AMD potential particularly with a change to the base case mine plan to ensure closure objectives in regard to AMD can be achieved. This includes updating the AMD CSM based on new information and the transition of AMD models from operations to closure to inform operational, closure and water management strategies.

- Verify and revise AMD management procedures to address any changes in understanding of AMD risk.

- Regular reporting of key monitoring parameters (leading and lagging) and key risk indicators. Regular reporting assists both routine regulatory reporting requirements as well as the real-time identification of developing trends.

- Review and document the design and operating effectiveness of AMD management controls on a risk-based frequency.

\section{$5 \quad$ Implementation strategy}

The Standard was released internally within BHP in March 2019. Implementation of the Standard will be undertaken utilising a phased and risk-based approach (noting that the requirements are minimum). Where situations exist that make compliance to the Standard problematic, an 'if-not, why-not' process will be utilised. An example of this will be on some of the closed legacy sites, where active exploration drilling programs are not taking place to facilitate the collection of data. In these situations, a tailored and fit-forpurpose approach will be required.

One of the first steps in the implementation of the Standard is for a site-by-site gap analysis to understand the current state of compliance with the Standard. A supplementary guidance note has also been developed to aid understanding and provide greater context and technical detail.

As management requirements become better understood, relevant operational level procedures may need to be developed to be compliant to the Standard.

Training modules have also been developed to improve understanding of AMD risks and provided to relevant disciplines that have the ability to influence the management of AMD, e.g. exploration geologists, hydrogeologists, mine planners and technical operational personnel (production supervisors, geologists and mining engineers).

The overall aspiration is that BHP have fully embedded management process to enable the AMD Strategy to be met, that is, proactively managing AMD materials to minimise the likelihood of in perpetuity management being required. 


\section{Conclusion}

AMD presents a key challenge to the successful closure of mines around the world. The BHP Global AMD Management Standard is a step change development that will enable global consistency in the characterisation and management of PAF materials across BHPs minerals portfolio. The Standard will ultimately drive AMD management to be seen as a business as usual, routine activity.

\section{References}

Commonwealth of Australia 2016a, Leading Practice Sustainable Development Program for the Mining Industry - Preventing Acid and Metalliferous Drainage, Canberra.

Commonwealth of Australia 2016b, Leading Practice Sustainable Development Program for the Mining Industry-Mine Rehabilitation, Canberra.

Department of Mines and Petroleum 2015, Guidelines for Preparing Mine Closure Plans, Perth.

Gasparon, M, Smedley, A, Jong, T, Costagliola, P \& Benvenuti, M 2007, 'Acid mine drainage at Mount Morgan, Queensland (Australia): experimental simulation and geochemical modelling of buffering reactions', in R Cidu \& F Frau (eds), Proceedings of the 2007 International Mine Water Association Symposium, Mako Edizioni, Cagliari.

INAP 2007, Global Acid Rock Drainage Guide (GARD Guide), International Network for Acid Prevention.

Lottermoser, BG 2010, Mine Wastes: Characterization, Treatment and Environmental Impacts, Springer, New York.

Miller, S 2014, 'Leading practice solutions for acid rock drainage prevention and control: key to achieving a sustainable future for mineral resource development', in H Miller \& L Preuss (eds), Proceedings of the Eighth Australian Workshop on Acid and Metalliferous Drainage, pp. 51-65.

Nordstrom, DK \& Alpers, CN 1999, 'Negative pH, efflorescent mineralogy, and consequences for environmental restoration at the Iron Mountain Superfund site, California', Proceedings of the National Academy of Sciences of the United States of America, vol. 96, pp. 3455-3462.

Pearce, Jl, Weber, PA, Pearce, SP \& Scott, P 2016a, 'Acid and metalliferous drainage contaminant load prediction for operational or legacy mines at closure', in AB Fourie \& M Tibbett (eds), Proceedings of the 11th International Conference on Mine Closure, Australian Centre for Geomechanics, Perth, pp. 663-676.

Pearce, SP, Lehane, S \& Pearce, Jl 2016b, 'Waste material placement options during construction and closure risk reduction quantifying the how, the why and the how much', in AB Fourie \& M Tibbett (eds), Proceedings of the 11th International Conference on Mine Closure, Australian Centre for Geomechanics, Perth, pp. 691-706.

Price, W 2009, Prediction Manual for Drainage Chemistry from Sulphidic Geologic Materials, Mine Environment Neutral Drainage (MEND) Program, Smithers, British Columbia.

Sernageomin 2015, Guia Metodologica para la Estabilidad Quimica de Faenas e Instalaciones, Chile.

Weber, PA, Malloch, K \& Kerr, G 2019, 'Geometallurgical tools to understand acid and metalliferous drainage risks for PACRIM mining projects', extended abstract presented at the AusIMM PACRIM Conference 2019, Auckland, 6 April 2019.

Wilson, W 2011, 'Rock dump hydrology: an overview of full-scale excavations and scale-up experiments conducted during the last two decades', in C Bell \& B Braddock (eds), Proceedings of the 7th Australian Workshop on Acid and Metalliferous Drainage, JK Tech, Indooroopilly, pp. 307-322. 
